\title{
The Mitochondria-Targeted Antioxidant SkQ1 Downregulates Aryl Hydrocarbon Receptor-Dependent Genes in the Retina of OXYS Rats with AMD-Like Retinopathy
}

\author{
M. L. Perepechaeva, ${ }^{1}$ A. Yu. Grishanova, ${ }^{1}$ E. A. Rudnitskaya, ${ }^{2}$ and N. G. Kolosova ${ }^{1,2,3}$ \\ ${ }^{1}$ Institute of Molecular Biology and Biophysics of Siberian Branch of RAMS, Timakova Street 2, Novosibirsk 630117, Russia \\ ${ }^{2}$ Institute of Cytology and Genetics, Prospekt Acad, Lavrentjeva 10, Novosibirsk 630090, Russia \\ ${ }^{3}$ Novosibirsk State University, Pirogova 2, Novosibirsk 630090, Russia \\ Correspondence should be addressed to M. L. Perepechaeva; perepech@niimbb.ru
}

Received 29 May 2014; Accepted 1 July 2014; Published 14 July 2014

Academic Editor: Miltiadis Tsilimbaris

Copyright ( 2014 M. L. Perepechaeva et al. This is an open access article distributed under the Creative Commons Attribution License, which permits unrestricted use, distribution, and reproduction in any medium, provided the original work is properly cited.

\begin{abstract}
The mitochondria-targeted antioxidant SkQ1 is a novel drug thought to retard development of age-related diseases. It has been shown that SkQ1 reduces clinical signs of retinopathy in senescence-accelerated OXYS rats, which are a known animal model of human age-related macular degeneration (AMD). The aim of this work was to test whether SkQ1 affects transcriptional activity of $A h R$ (aryl hydrocarbon receptor) and Nrf2 (nuclear factor erythroid 2-related factor 2), which are considered as AMD-associated genes in the retina of OXYS and Wistar rats. Our results showed that only $A h R$ and $A h R$-dependent genes were sensitive to SkQ1. Dietary supplementation with SkQ1 decreased the AhR mRNA level in both OXYS and Wistar rats. At baseline, the retinal Cyp1a1 mRNA level was lower in OXYS rats. SkQ1 supplementation decreased the Cypla1 mRNA level in Wistar rats, but this level remained unchanged in OXYS rats. Baseline Cyp1a2 and Cyp1b1 mRNA expression was stronger in OXYS than in Wistar rats. In the OXYS strain, Cyp1a2 and Cyp1b1 mRNA levels decreased as a result of SkQ1 supplementation. These data suggest that the Cypla2 and Cyplb1 enzymes are involved in the pathogenesis of AMD-like retinopathy of OXYS rats and are possible therapeutic targets of SkQ1.
\end{abstract}

\section{Introduction}

Mitochondria-targeted antioxidant SkQ1 (cationic plastoquinone derivative (10-[6'-plastoquinonyl] decyltriphenylphosphonium) is a novel medication that is designed to retard the development of age-related diseases and aging $[1,2]$. Plastoquinone, a very effective electron carrier and an antioxidant of chloroplasts, was conjugated with decyltriphenylphosphonium to obtain a cation that easily penetrates the cell membrane [3-5]. SkQ1's geroprotective properties are based on the ability of this reagent to attenuate pathological processes associated with production of reactive oxygen species [4]. The effects of SkQ1 on aging are accompanied by inhibition of development of such age-related problems as cataract, retinopathy, glaucoma, balding, canities, osteoporosis, involution of the thymus, and peroxidation of lipids and proteins $[1,3]$. Recently it was shown that addition of SkQ1 to food or treatment with SkQ1 eye drops not only prevents development of retinopathy but also reduces severity of preexisting pathological changes in the retina of senescenceaccelerated OXYS rats [6].

OXYS rats are a known animal model of human agerelated macular degeneration (AMD) $[7,8]$. OXYS rats develop a retinopathy similar to the dry form of human AMD judging by the symptoms, morphology, and some molecular changes. The OXYS retinopathy involves hypoplasia and atrophy of the retinal pigment epithelium and of photoreceptors, formation of drusen, and retinal neovascularization; this retinopathy also correlates with expression of VEGF (vascular endothelial growth factor) [8]. It was reported that the effects of SkQ1 include improvement of functions of the retinal pigment epithelium and a reduction of lipofuscin accumulation 
in the OXYS retina $[9,10]$. The molecular mechanisms underlying the effects of SkQ1 have yet to be investigated.

AMD etiology includes genetic predisposition, exposure to environmental toxins and free radicals, and low levels of antioxidants. The pathogenesis of AMD is being actively studied but is not fully understood at present; several key factors are known to be involved. Oxidative stress is one of these key factors together with structural and functional changes in the retinal pigment epithelium, inflammation, and activation of the complement cascade. Recently, some genes that take part in the regulation of redox processes were characterized as possible AMD-associated genes. These genes include nuclear factor erythroid 2-related factor 2 (Nrf2) [11] and aryl hydrocarbon receptor (AhR) [12]. After analysis of haplotypes of detoxification genes, $A h R$ caught the attention of researchers as a possible risk factor of AMD [12]. Studies of $A h R^{-/-}$mice further support the potential role of this gene in AMD pathogenesis [13].

AhR is a ligand-dependent transcription factor that recognizes and binds to a wide range of xenobiotics and endogenous compounds. When inactive, AhR is located in the cytoplasm along with its associated proteins. After AhR binds to its ligand, the above-mentioned complex disintegrates, ligand-bound AhR moves to the nucleus, and dimerizes with Arnt (aryl hydrocarbon receptor nuclear translocator). The AhR/Arnt heterodimer then binds to xenobiotic-responsive elements (XREs) in the genomic DNA, and this process causes initiation of transcription of AhR's target genes [14,15].

AhR controls expression of some components of phase I and phase II of xenobiotic metabolism [14, 15]. The following are phase I enzymes: cytochrome P450 1 subfamily (Cypla1, Cypla2, and Cyplb1) and aldehyde dehydrogenase 3A1 (Aldh3al) as a participant of phase II of the xenobiotic metabolism [16].

$\mathrm{Nrf} 2$ is a transcription factor that controls gene expression of antioxidant systems of the cell. In particular, it controls expression of heme oxygenase 1 (Hmoxl), thioredoxin reductase 1 (Txnrd1), and glutathione S-reductase (Gsr) [17]. Nrf2, while in a complex with Maf proteins, interacts with antioxidant-responsive elements (ARE) in the promoter region of target genes [18].

Recent findings demonstrate a relationship between AhRdependent and Nrf2-dependent signal transduction pathways; Nrf2 may be a genomic target of AhR, and the possibility of a cross-talk between the AhR/XRE and Nrf2/ARE signal transduction pathways cannot be ruled out $[16,19]$. Some genes are controlled by both AhR and Nrf2. The list includes $\mathrm{NAD}(\mathrm{P}) \mathrm{H}$ : quinone oxidoreductase 1 (Nqo1), uracil diphosphate- (UDP-) glucuronosyltransferase 1A6 (Ugt1a6), UDP-glucuronosyltransferase (UGT) 1A9 (Ugt1a9), glutathione S-transferase (GST) A1 (Gsta1), and a number of other isoforms of UGT and GST [16]. Genes that are controlled by AhR, Nrf2, and both AhR and Nrf2 are sometimes called the AhR-Nrf2 gene battery.

The aim of this study was to test whether the antioxidant SkQ1 affects transcriptional activity of a redox-sensitive system: the AhR-Nrf2 gene battery. OXYS and Wistar rats received SkQ1 with food between the ages of 1.5 to 3 months, which is the period of active manifestation of signs of retinopathy. It was repeatedly proven previously that SkQ1 in this regimen can prevent the development of retinopathy in OXYS rats $[6,20]$.

\section{Methods}

2.1. Reagents. TRI-Reagent and RNA Secure Reagent were purchased from Ambion (USA); the cDNA synthesis MMLV RT kit and PCR kit qPCRmix-HS were from Evrogen (Russia); RNasin and RQ1 DNase were from Promega (USA); oligonucleotides (primers) for analysis of the rat genes Cyp1a1, Cyp1a2, Cyp1b1, Gsta1, Nqo1, Aldh3a1, Ugt1a6, Ugt1a9, AhR, Nrf2, Gsr, Txnrd1, Hmox1, and Gapdh were from Syntol (Russia). SkQ1 was synthesized as described earlier [5]. All other chemicals were obtained from other commercial sources and were analytical grade.

2.2. Animals. Male senescence-accelerated OXYS and agematched male Wistar (control) rats were obtained from the Shared Center for Genetic Resources of Laboratory Animals of the Institute of Cytology and Genetics, Siberian Branch of the Russian Academy of Sciences (SB RAS; Novosibirsk, Russia). The rats were kept under standard laboratory conditions (at $22 \pm 2{ }^{\circ} \mathrm{C}, 60 \%$ relative humidity, and natural light), provided with standard rodent feed, PK-120-1, Ltd. (Laboratorsnab, Russia), and given water ad libitum. All experiments in this study were approved by the Institutional Review Board and performed in accordance with the Animal Care Regulations of the Institute of Cytology and Genetics (Novosibirsk) and with the international norms for studies on laboratory animals.

To assess the effects of SkQ1 (from the age of 1.5 months to the age of 3 months) on gene expression, 1.5-month-old male OXYS rats were randomly assigned to 1 of the 2 groups: the standard (control) diet or the diet supplemented with 250 nmol SkQ1 per kilogram of body weight per day (15 rats per group). The age-matched Wistar rats (standard diet) served as a control (15 rats in this group). The rats were euthanized using $\mathrm{CO}_{2}$ inhalation and killed by decapitation 5 days after the last examination of eyes. The retinas were removed, frozen, and stored at $-80^{\circ} \mathrm{C}$ until analysis.

2.3. RNA Isolation and Reverse Transcription. Total RNA was isolated using the TRI-Reagent isolation kit (Ambion) as per the manufacturer's protocol. The RNA pellets were dissolved in $1 \mathrm{mM}$ sodium citrate buffer $\mathrm{pH} 6.5$, containing $1 \times \mathrm{RNA}$ Secure Reagent (Ambion). The RNA concentration was measured using UV spectrophotometry. The RNA samples were treated with RNase-free DNase (Promega, USA) according to the manufacturer's instructions. Then the samples were subjected to repeated RNA extraction with a phenol-chloroform mixture and pure chloroform followed by precipitation with propanol. Reverse transcription was performed using the cDNA synthesis MMLV RT kit (Evrogen, Russia) according to the manufacturer's protocol. 
TAble 1: Primer sequences for analysis of gene expression.

\begin{tabular}{|c|c|c|}
\hline Gene & & Sequence \\
\hline \multirow{3}{*}{ Cypla1 } & Forward & $5^{\prime}$-CCAAACGAGTTCCGGCCT-3' \\
\hline & Reverse & $5^{\prime}$-TGCCCAAACCAAAGAGAATGA-3' \\
\hline & Probe & 5'(FAM)-TTCTCACTCAGGTGTTTGTCCAGAGTGCC-(BHQ1)3' \\
\hline \multirow{3}{*}{ Cypla2 } & Forward & $5^{\prime}$-CGCCCAGAGCGGTTTCTTA-3' \\
\hline & Reverse & $5^{\prime}$-TCCCAAGCCGAAGAGCATC-3' \\
\hline & Probe & 5'(FAM)-CAATGACAACACGGCCATCGACAAG-(BHQ1)3' \\
\hline \multirow{3}{*}{ Cyplb1 } & Forward & $5^{\prime}$-GGCATCGCACTTGTACTTCG-3' \\
\hline & Reverse & $5^{\prime}$-CACCAGAGCCTGATGGATGG-3' \\
\hline & Probe & $5^{\prime}(\mathrm{FAM})$-TCTCGCCATTCAGCACCACCACGG-(BHQ1)3' \\
\hline \multirow{3}{*}{ Gstal } & Forward & $5^{\prime}$-ACTACATTGCCACCAAATACAACCT-3' \\
\hline & Reverse & $5^{\prime}$-CACTCCTTCTGCATACATGTCGAT-3' \\
\hline & Probe & $5^{\prime}$ (FAM)-ATGGGAAGGACATGAAGGAGAGAGCCC-(BHQ1)3' \\
\hline \multirow{3}{*}{ Nqo1 } & Forward & 5'-TTGAGTCATCTCTGGCGTATAAGG-3' \\
\hline & Reverse & $5^{\prime}$-GGTCTGCAGCTTCCAGCTTT-3' \\
\hline & Probe & $5^{\prime}$ (FAM)-AGGCCGCCTGAGCCCGGATA-(BHQ1)3' \\
\hline \multirow{3}{*}{ Aldh3a1 } & Forward & $5^{\prime}$-CCGTGATTATGGGAGGATCATC-3' \\
\hline & Reverse & $5^{\prime}$-TGGGCTACTTTCTGGTTGTCAAT-3' \\
\hline & Probe & $5^{\prime}$ (FAM)-TGACCGTCACTTCCAGCGGGTCA-(BHQ1)3' \\
\hline \multirow{3}{*}{ Ugt1a6 } & Forward & $5^{\prime}$-CCTTGGACGTGATTGGCTTT-3' \\
\hline & Reverse & $5^{\prime}$-GCAGCCATAGGCACAACTTTTATA-3' \\
\hline & Probe & 5'(FAM)-CTGGCCATCGTGTTGACGGTGGT-(BHQ1)3' \\
\hline \multirow{3}{*}{ Ugt1a9 } & Forward & $5^{\prime}$-GAGGCTTTGGGCAGAATTCC-3' \\
\hline & Reverse & $5^{\prime}$-TTTGCAAGGTTCGATGGTCTAGTT-3' \\
\hline & Probe & $5^{\prime}$ (FAM)-CAGACGGTCCTGTGGCGCTACACC-(BHQ1)3' \\
\hline \multirow{3}{*}{$A h R$} & Forward & 5' -TGGACAAACTCTCCGTTCTAAGG-3' \\
\hline & Reverse & $5^{\prime}$-GATTTTAATGCAACATCAAAGAAGCT-3' \\
\hline & Probe & 5'(FAM)-CAGCGTCACGTACCTGAGGGCCA-(BHQ1)3' \\
\hline \multirow{3}{*}{$N r f 2$} & Forward & $5^{\prime}$-AGCAACTCCAGAAGGAACAGGAGA-3' \\
\hline & Reverse & 5'-CTTGTTTGGGAATGTGGGCAACCT-3' \\
\hline & Probe & $5^{\prime}(\mathrm{FAM})$-TCCCAATTCAGCCAGCCCAGCACA-(BHQ1) $3^{\prime}$ \\
\hline \multirow{3}{*}{ Hmoxl } & Forward & $5^{\prime}$-TTACACACCAGCCACACAGCACTA- $3^{\prime}$ \\
\hline & Reverse & 5'-CATGGCCTTCTGCGCAATCTTCTT-3' \\
\hline & Probe & $5^{\prime}$ (FAM)-FAMTGAGCTGCTGGTGGCCCACGCATATA-(BHQ1) $3^{\prime}$ \\
\hline \multirow{3}{*}{ Txnrd1 } & Forward & $5^{\prime}$-TTTACTCAGCAGAGCGGTTCCT-3' \\
\hline & Reverse & $5^{\prime}$-TGCACATTCCAAGGCGACAT-3' \\
\hline & Probe & $5^{\prime}$ (FAM)-AAGACCCTAGTGGTTGGCGCGTCCT-(BHQ1)3' \\
\hline \multirow{3}{*}{ Gsr } & Forward & $5^{\prime}$-CTTCGACAATACGGTCGCCATTCA-3' \\
\hline & Reverse & $5^{\prime}$-AATCTATAAAGCTGGCGCAGGACG-3' \\
\hline & Probe & 5'(FAM)-AGTGGGCCTCTGGGAGGAACCAATCA-(BHQ1)3' \\
\hline \multirow{3}{*}{ Gapdh } & Forward & $5^{\prime}$-CAAGGTCATCCATGACAACTTTG- $3^{\prime}$ \\
\hline & Reverse & $5^{\prime}$-GGGCCATCCACAGTCTTCTG-3' \\
\hline & Probe & $5^{\prime}$ (FAM)-ACCACAGTCCATGCCATCACTGCCA-(BHQ1)3' \\
\hline
\end{tabular}

2.4. Real-Time PCR. The PCR primer sequences used are presented in Table 1.

Gapdh served as an internal control (housekeeping gene). The gene expression patterns were analyzed using the iCycler CFX96 real-time PCR detection system (Bio-Rad Laboratories, USA) based on the TaqMan principle. Aliquots from all cDNA samples were mixed, and the "average" solution was used for preparation of calibration curves, which were used for measurement of relative cDNA levels of genes under study and of a reference gene in experimental samples. The reaction mixture contained the qPCRmix-HS buffer (Evrogen, Russia); a primer mix consisting of $0.5 \mu \mathrm{L}$ of $5 \mu \mathrm{M}$ probe, $1 \mu \mathrm{L}$ of a $10 \mu \mathrm{M}$ forward primer, and $1 \mu \mathrm{L}$ of a $10 \mu \mathrm{M}$ reverse primer; and $2000 \mathrm{ng}$ cDNA. The reaction was conducted under 
the following conditions: heating at $95^{\circ} \mathrm{C}$ for $3 \mathrm{~min}$, then 40 cycles of denaturation at $95^{\circ} \mathrm{C}$ for $15 \mathrm{~s}$, and annealing/extension at $60^{\circ} \mathrm{C}$ for $30 \mathrm{~s}$.

In each experiment, we added samples of cDNA under study with primers specific to a target gene (in triplicate for each cDNA sample) to wells of 1 multiwell plate, and similar samples with primers specific to a comparison gene were added to other wells of the same multiwell plate (also in triplicate). From these cDNA samples, we took identical amounts of cDNA to build a standard curve (this was an absolute quantification method using a standard curve). We used serial dilutions of the standard cDNA from $1: 3$ to $1: 27$. To wells of 1 multiwell plate, we added 2-3 repeats of reactions containing primers specific to a target gene and similar samples with primers specific to a comparison gene (2-3 repeats). Using the resulting standard curves, we quantified the original amount of cDNA (relative to the standard cDNA), and this value was normalized to the amount of cDNA of the comparison gene (Gapdh) [21]. For each cDNA sample, PCR was repeated at least twice.

2.5. Statistical Analysis. All calculations were performed using the STATISTICA software package (StatSoft, Inc., USA). All data were analyzed using two-way ANOVA and the Newman-Keuls post hoc test. The independent variables were genotype (Wistar, OXYS) and treatment (controls, SkQ1). One-way ANOVA was used for individual group comparison. The data are presented as mean \pm SEM. The results were considered statistically significant if the $P$ value was less than 0.05 .

\section{Results}

In this work, we analyzed mRNA expression of AhR and AhR-dependent genes, Nrf2 and Nrf2-dependent genes, and AhR+Nrf2-dependent genes in phases I and II of xenobiotic metabolism.

Figure 1 shows mRNA levels of AhR and AhR-dependent genes of phase I (Cyp1a1, Cypla2, and Cyp1b1) and phase II (Aldh3a1). Our data show that the genotype had no influence on the $A h R$ mRNA level (Figure $1(\mathrm{a}))$ in the retina $\left(F_{1,22}=\right.$ $1.02, P=0.32)$, but this parameter was affected by SkQ1 $\left(F_{1,22}=16.00, P=0.0007\right)$. SkQ1 supplementation decreased the $A h R$ mRNA level both in OXYS rats (1.9-fold; $P<0.012)$ and in Wistar rats (1.7-fold; $P<0.17)$.

At the same time, the retinal Cypla1 (Figure 1(b)), Cypla2 (Figure 1(d)), and Cyp1b1 (Figure 1(e)) mRNA level was affected by genotype $\left(F_{1,26}=7.54, P=0.011 ; F_{1,24}=6.69, P=\right.$ $0.016 ; F_{1,25}=4.43, P=0.047$, resp.). Post hoc analysis showed that the retinal Cyplal mRNA level (Figure 1(b)) was $~ 50 \%$ lower in OXYS rats than the Wistar strain $(P=0.01)$. Cypla2 (Figure 1(d)) and Cyp1b1 (Figure 1(e)) mRNA expression was 2 -fold $(P=0.03)$ and 1.7 -fold $(P=0.02)$ higher in OXYS than in Wistar rats, respectively.

SkQ1 supplementation downregulated only Cyp1a1 mRNA expression (2-fold; $P=0.03$ ) in the retina of Wistar rats (Figure 1(b)), whereas in the OXYS retina, the Cypla1 mRNA expression remained unchanged (Figure 1(b)). In the retina of OXYS rats, the Cypla2 (Figure 1(d)) and Cyp1b1 (Figure 1(e)) mRNA levels were decreased 2.2-fold $(P=0.03)$ and 1.7-fold $(P=0.02)$, respectively, as a result of the dietary SkQ1 supplementation. Cyp1a2 (Figure 1(d)) and Cyp1b1 (Figure 1(e)) mRNA expression remained unchanged in Wistar rats between SkQ1 supplementation and control.

According to two-way ANOVA there were no statistically significant differences in the mRNA expression of Aldh3al (Figure 1(c)) either between the 2 strains $\left(F_{1,25}=3.7, P=0.07\right)$ or between SkQ1 supplementation and control $\left(F_{1,25}=2.5\right.$, $P=0.13)$. There were no statistically significant differences in the mRNA expression of Aldh3al (Figure 1(c)) either between the 2 strains or between SkQ1 supplementation and control.

Figure 2 shows mRNA levels of genes controlled by both AhR and Nrf2: Nqo1, Gsta1, Ugt1a6, and Ugt1a9. Two-way ANOVA showed that Nqo1 mRNA level (Figure 2(a)) was affected by genotype $\left(F_{1,26}=4.48, P=0.04\right)$ but was not affected by SkQ1 supplementation $\left(F_{1,26}=1.67, P=0.21\right)$. One-way ANOVA showed a decreased Nqo1 mRNA level in the retina of control Wistar rats compared to OXYS rats (1.9fold; $P=0.02$ ).

There were no statistically significant differences in the mRNA level of Ugtla6 (Figure 2(c)), Gstal (Figure 2(b)), and Ugt1a9 (Figure 2(d)) between the 2 strains $\left(F_{1,25}=0.25, P=\right.$ $0.62 ; F_{1,26}=2.75, P=0.11$; and $F_{1.26}=0.56, P=0.47$, resp. $)$ or between SkQ1 supplementation and control $\left(F_{1,25}=3.8\right.$, $P=0.06 ; F_{1,26}=0.54, P=0.47$; and $F_{1,26}=0.01, P=0.92$, resp.).

Figure 3 shows mRNA levels of Nrf2 and Nrf2-dependent genes: Gsr, Hmoxl, and Txnrd1. There were no statistically significant differences in the mRNA level of Nrf2 (Figure 3(a)) and of Nrf2-dependent genes Gsr (Figure 3(b)) and Hmox1 (Figure 3(d)) either between the 2 strains $\left(F_{1,26}=0.77, P=\right.$ $0.39 ; F_{1,26}=1.18, P=0.29$; and $F_{1,24}=3.57, P=0.07$, resp.) or between SkQ1 supplementation and control $\left(F_{1,26}=0.001\right.$, $P=0.997 ; F_{1,26}=1.42, P=0.24$; and $F_{1,26}=0.17, P=0.68$, resp.).

Two-way ANOVA analysis showed that the mRNA level of the Nrf2-dependent gene Txrnd1 (Figure 3(c)) was not affected by genotype $\left(F_{1,26}=1.65, P=0.21\right)$ but was affected by SkQ1 supplementation $\left(F_{1,26}=5.55, P=0.026\right)$. One-way ANOVA revealed a difference between the level of Txrnd1 (Figure 3(c)) in the retina of untreated Wistar and OXYS rats (1.5-fold; $\left.F_{1,12}=13.5, P=0.003\right)$. SkQ1 supplementation downregulated Txrnd1 mRNA expression (1.7-fold; $F_{1,13}=$ $5.85, P=0.03)$ in the retina of OXYS rats, whereas in the Wistar retina, the Txrnd1 mRNA expression remained unchanged $\left(F_{1,13}=0.39, P=0.54\right)$.

\section{Discussion}

The retina is among the types of tissue that are at high risk of damage by reactive oxygen species, whereas oxidative stress is a major contributor to the pathogenesis of retinopathy and AMD [22]. Our previous research showed that the mitochondria-targeted antioxidant SkQ1 can reduce clinical manifestations of retinopathy in OXYS rats. Nonetheless, the molecular mechanisms behind SkQ1's beneficial 


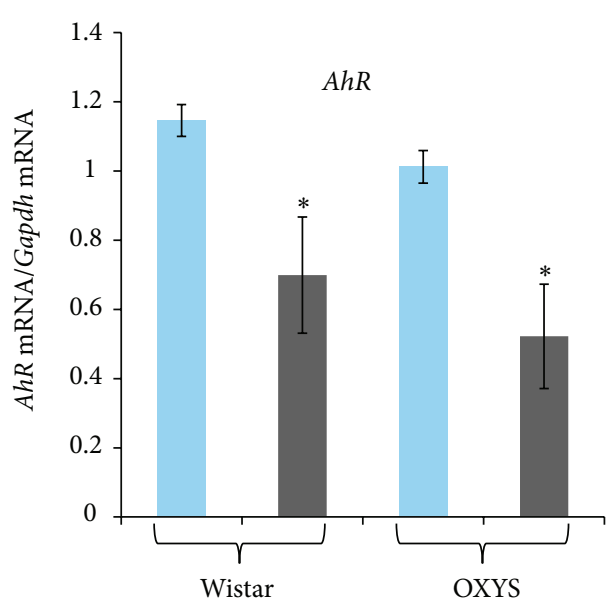

(a)

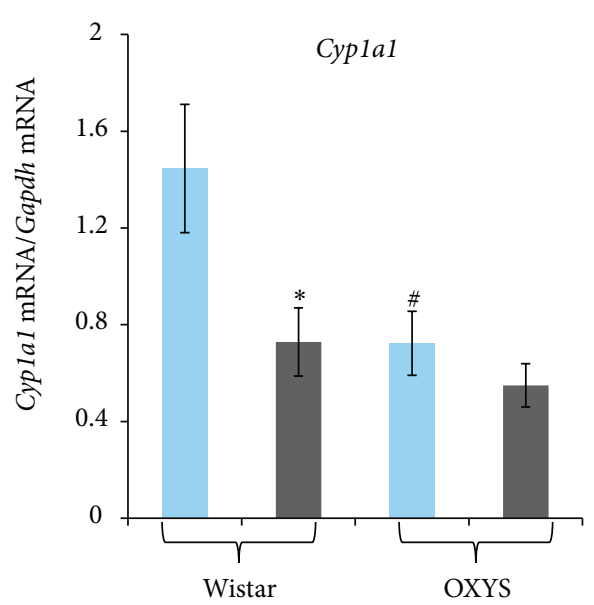

(b)

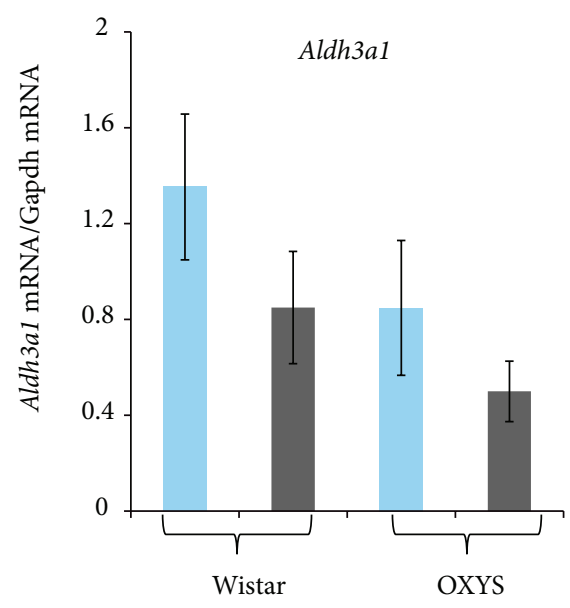

(c)

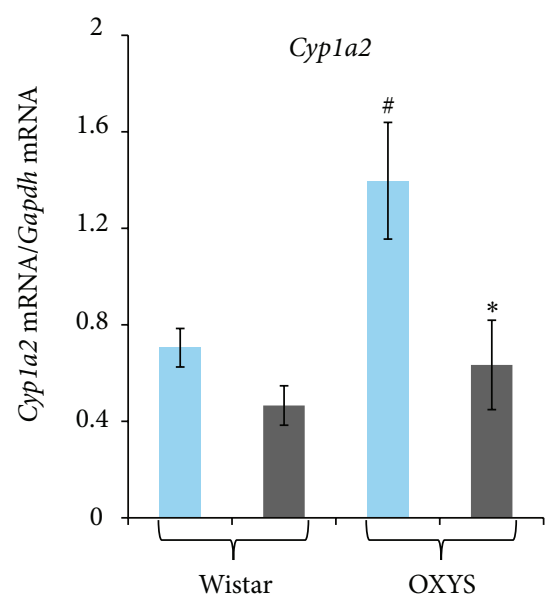

(d)

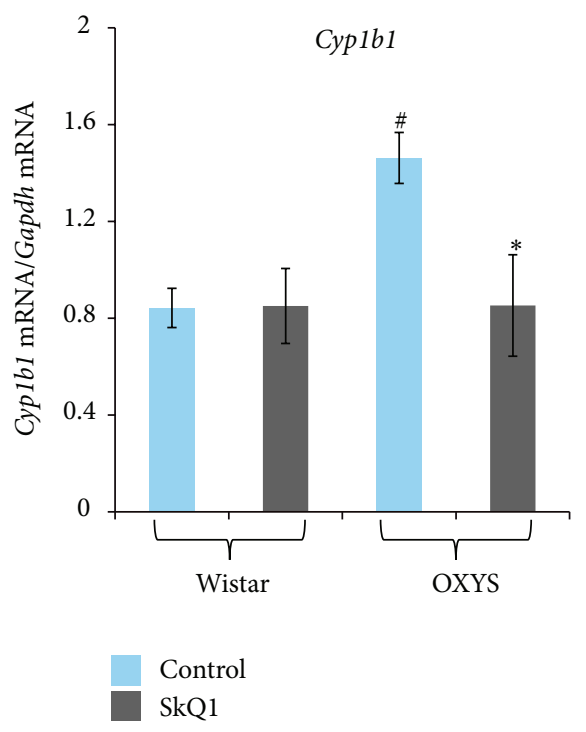

(e)

FIgure 1: AhR (a), Cypla1 (b), Aldh3a1 (c), Cypla2 (d), and Cyp1b1 (e) mRNA levels in the retinas of 3-month-old Wistar and OXYS rats treated with SkQ1. All data are normalized to the expression level of a housekeeping gene (Gapdh). Values are presented as mean \pm SEM ( $n=6$ to 8). Significant differences between groups are marked with ${ }^{*} P<0.05$.

effects are not well understood. Using an animal model of retinopathy-OXYS rats-in the present work, we explored the influence of SkQ1 on mRNA expression of $A h R, N r f 2$, and their dependent genes; those genes can regulate oxidative and antioxidant processes in the cells.

Our present data suggest that only $A h R$ and $A h R$ dependent genes of phase I of xenobiotic biotransformation were strongly sensitive to SkQ1 supplementation. The decrease of the Ugt1a6 mRNA level and low Nqo1 mRNA expression in OXYS rats (compared to the Wistar strain) can be explained in part by $\mathrm{AhR}$ dependence of these genes. The observed effect of SkQ1 on Txrnd1 mRNA expression was a surprise because direct links between Txrndl and AhR are unknown, whereas Nrf2 and Nrf2-dependent genes Gsr and Hmoxl were not affected. The absence of effects of the antioxidant $\mathrm{SkQ1}$ on the genes responsible for major endogenous antioxidant systems suggests that the antioxidant activity of SkQ1 is not linked to the effects of SkQ1 on the transcription factor Nrf2 or on enzymes of phase 2 of the xenobiotic metabolism. This is not surprising; the mechanism of action of SkQ1 does not imply involvement of transcription factors or regulation of gene expression in general.

Nonetheless, we see the effects of SkQ1 on the mRNA expression of the transcription factor AhR and on mRNA levels of genes of phase I metabolism of xenobiotics that are activated by AhR. These findings are supported indirectly by the data on the influence of SkQ1 on P450 cytochrome activity in the rat liver (Grishanova et al., unpublished observations).

Differences in the level of mRNA of AhR-dependent genes are observed not only under the influence of SkQ1 but also between untreated Wistar and OXYS rats. For example, the observed level of Cyplal mRNA is lower in OXYS 


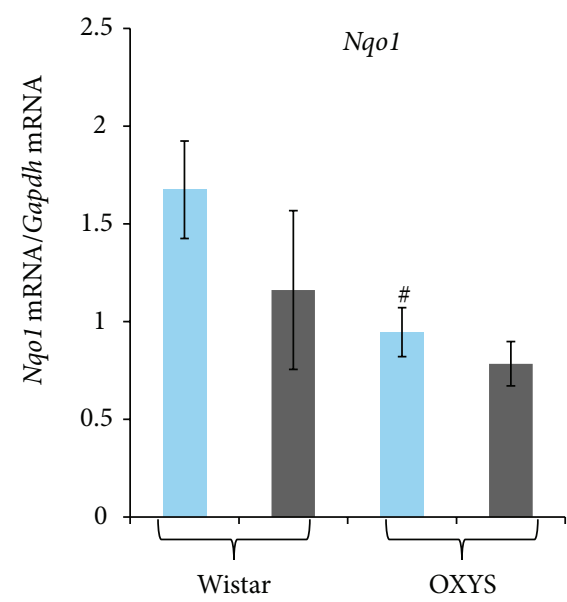

(a)

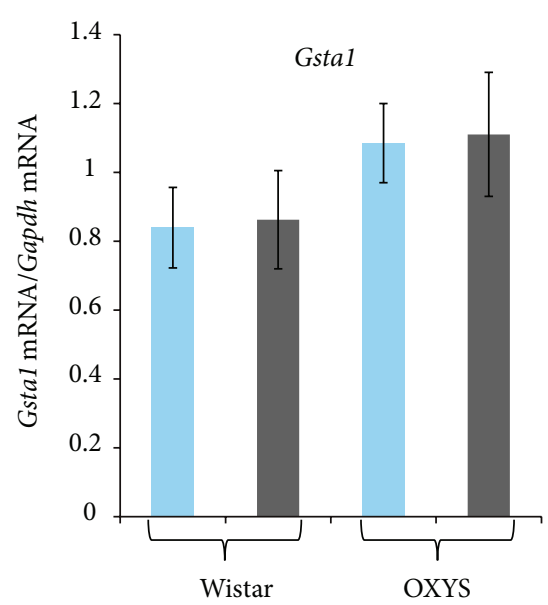

(b)

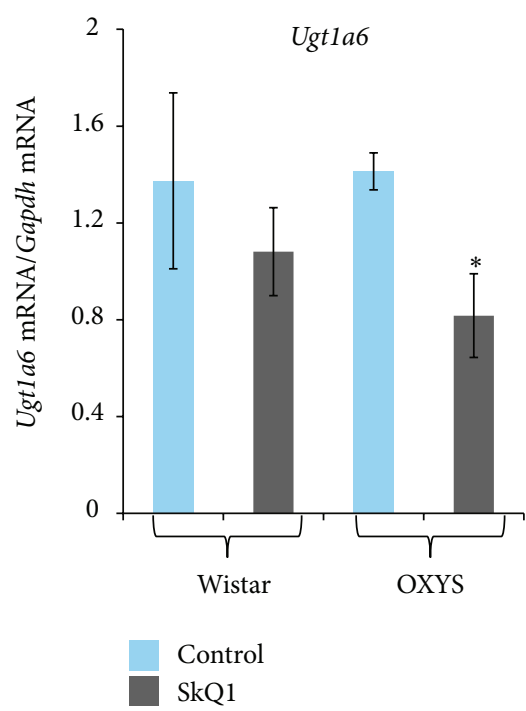

(c)

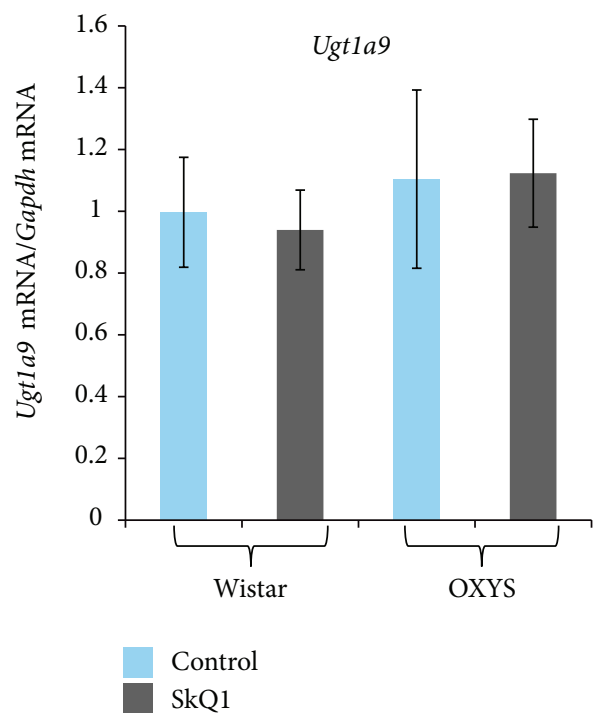

(d)

Figure 2: Nrqo1 (a), GstA1 (b), Ugt1a6 (c), and Ugt1a9 (d) mRNA levels in the retinas of 3-month-old Wistar and OXYS rats treated with SkQ1. All data are normalized to the expression level of a housekeeping gene (Gapdh). Values are presented as mean \pm SEM ( $n=6$ to 8). Significant differences between groups are marked with ${ }^{*} P<0.05$.

rats than the Wistar strain. This situation can be explained by the significant decrease in Cypla1 expression under oxidative stress [23], which constitutes one of the stages of the pathogenesis of AMD and AMD-like retinopathy in OXYS rats.

On the other hand, at baseline, Cyp1a2 and Cyp1b1 mRNA levels are higher in OXYS than in Wistar rats. It is known that some P450 cytochromes can metabolize arachidonic acid to compounds that affect the tone of a vessel wall and arterial blood pressure [24-26]. In particular, Cyp1b1 participates in the synthesis of 12-HETE (12-hydroxyeicosatetraenoic acid), which is known to be cardiotoxic [27]. Arachidonic acid can be metabolized by Cypla2 [28]; as a result, production of epoxyeicosatrienoic acids is enhanced. The latter compounds can serve as a source of reactive oxygen species in addition to being vasodilators and proangiogenic factors able to stimulate growth of endothelial and mesangial cells [29].

Retinopathy pathogenesis includes pathological neovascularization. It has been shown that several metabolites of arachidonic acid can activate the above process. For example, cytosolic phospholipase $\mathrm{A}(2)$ has proangiogenic properties and stimulates pathological retinal angiogenesis [30]. Furthermore, retinal neovascularization is associated with increased 12-lipoxygenase expression and with enhanced production of 12-HETE, 15-HETE, and 5-HETE [31]. Accordingly, the elevated Cyp1a2 and Cyp1b1 mRNA level in OXYS rats compared to the Wistar strain is likely to reflect (at least partially) the process of pathological neovascularization in OXYS rats. 


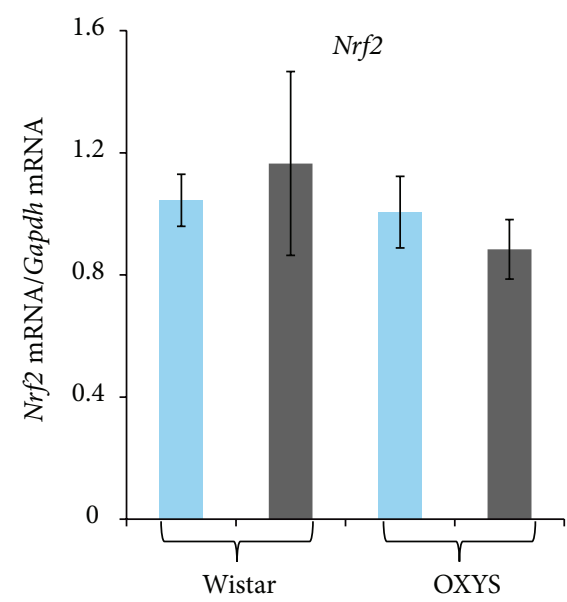

(a)

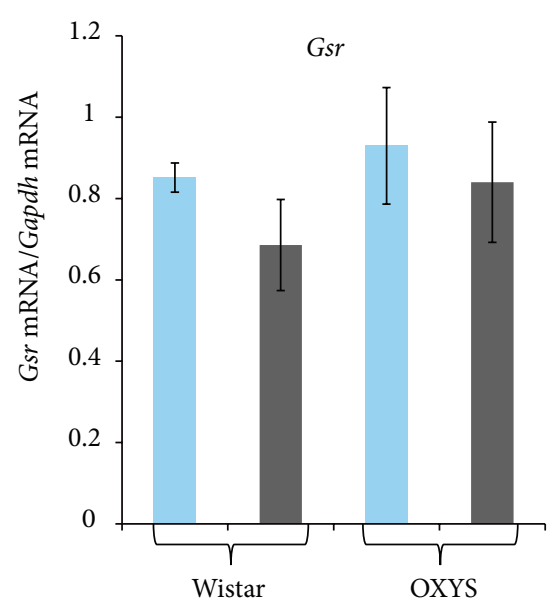

(b)

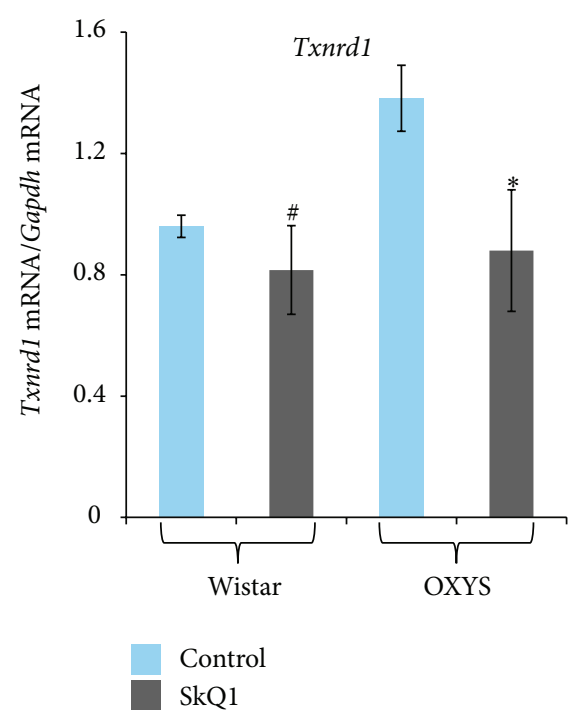

(c)

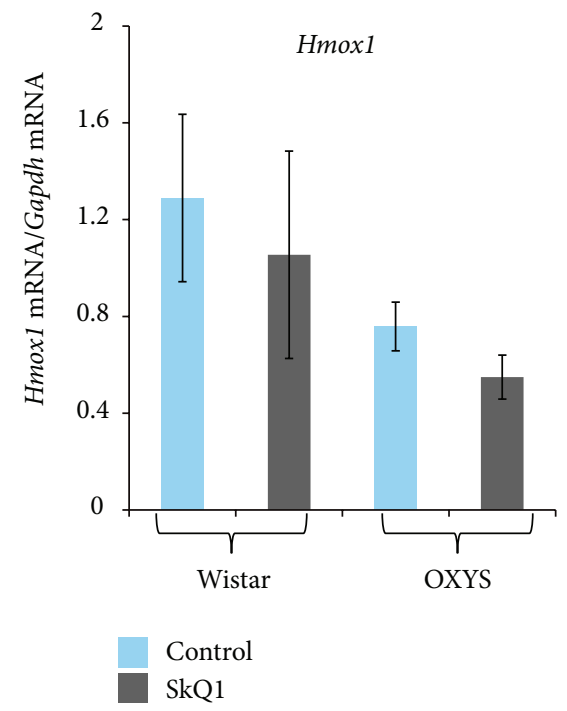

(d)

FIGURE 3: Nrf2 (a), Gsr (b), Txnrd1 (c), and Hmox1 (d) mRNA levels in the retinas of 3-month-old Wistar and OXYS rats treated with SkQ1. All data are normalized to the expression level of a housekeeping gene (Gapdh). Values are presented as mean \pm SEM $(n=6$ to 8$)$. Significant differences between groups are marked with ${ }^{*} P<0.05$.

In this work, the effects of SkQ1 supplementationwhere they are present-consist of downregulation of mRNA expression of the relevant genes. First, it is the reduced mRNA level of AhR both in OXYS and in Wistar rats. It is possible that the reduction in mRNA expression of AhR-controlled genes (Cypla1, Cypla2, and Cyp1b1) and the partially AhR-controlled gene Ugtla6 is mediated by SkQ1's influence on functioning of the AhR enzyme. Nonetheless, it is of course impossible to rule out direct action on the protein molecules of P450 cytochromes. Undoubtedly, detailed elucidation of such a mechanism of action of SkQ1 would be interesting, and further research is needed.

\section{Conclusion}

Our results point to the involvement of Cypla2 and Cyp1b1 in the pathogenesis of AMD-like retinopathy in OXYS rats. Cypla2 and Cyplb1 can be considered possible therapeutic targets for novel treatments of AMD; it is plausible that these enzymes are targets of SkQ1 when it is administered systemically.

\section{Abbreviations}

AhR: Aryl hydrocarbon receptor Aldh3a1: Aldehyde dehydrogenase 3A1 
AMD: Age-related macular degeneration

ARE: Antioxidant-responsive elements

Arnt: Ah receptor nuclear translocator

BHQ: Black hole quencher

Cyplal: Cytochrome P450 1A1

Cypla2: Cytochrome P450 1A2

Cyplb1: Cytochrome P450 1B1

FAM: 6-Carboxyfluorescein

Gsr: Glutathione-S-reductase

Gstal: Glutathione S-transferase A1

Hmoxl: Heme oxygenase 1

Nqo1: NADPH-quinone oxidoreductase

Nrf2: Nuclear factor, erythroid derived 2

Txnrd1: Thioredoxin reductase 1

Ugtla6: UDP-glucuronosyltransferase 1A6

Ugtla9: UDP-glucuronosyltransferase 1A9

VEGF: Vascular endothelium growth factor

XRE: Xenobiotic responsive element.

\section{Conflict of Interests}

The authors declare that there is no conflict of interests regarding the publication of this paper.

\section{Acknowledgment}

This work was supported by the Russian Foundation for Basic Research (Project no. 12-04-01352-a).

\section{References}

[1] V. P. Skulachev, Y. N. Anisimov, Y. N. Antonenko et al., "An attempt to prevent senescence: a mitochondrial approach," Biochimica et Biophysica Acta, vol. 1787, no. 5, pp. 437-461, 2009.

[2] V. P. Skulachev, "Cationic antioxidants as a powerful tool against mitochondrial oxidative stress," Biochemical and Biophysical Research Communications, vol. 441, no. 2, pp. 275-279, 2013.

[3] M. V. Skulachev, Y. N. Antonenko, V. N. Anisimov et al., "Mitochondrial-targeted plastoquinone derivatives: effect on senescence and acute age-related pathologies," Current Drug Targets, vol. 12, no. 6, pp. 800-826, 2011.

[4] L. E. Bakeeva, I. V. Barskov, M. V. Egorov et al., "Mitochondriatargeted plastoquinone derivatives as tools to interrupt execution of the aging program. 2. Treatment of some ROS- and agerelated diseases (heart arrhythmia, heart infarctions, kidney ischemia, and stroke)," Biochemistry, vol. 73, no. 12, pp. 12881299, 2008.

[5] Y. N. Antonenko, A. V. Avetisyan, L. E. Bakeeva et al., "Mitochondria-targeted plastoquinone derivatives as tools to interrupt execution of the aging program. 1. Cationic plastoquinone derivatives: synthesis and in vitro studies," Biochemistry, vol. 73, no. 12, pp. 1273-1287, 2008.

[6] A. M. Markovets, A. Z. Fursova, and N. G. Kolosova, "Therapeutic action of the mitochondria-targeted antioxidant SkQ1 on retinopathy in OXYS rats linked with improvement of VEGF and PEDF gene expression," PLoS ONE, vol. 6, no. 7, Article ID e21682, 2011.
[7] M. E. Pennesi, M. Neuringer, and R. J. Courtney, "Animal models of age related macular degeneration," Molecular Aspects of Medicine, vol. 33, no. 4, pp. 487-509, 2012.

[8] A. M. Markovets, V. B. Saprunova, A. A. Zhdankina, A. Z. Fursova, L. E. Bakeeva, and N. G. Kolosova, "Alterations of retinal pigment epithelium cause AMD-like retinopathy in senescentaccelerated OXYS rats," Aging, vol. 3, no. 1, pp. 44-54, 2011.

[9] V. B. Saprunova, D. I. Pilipenko, A. V. Alexeevsky, A. Z. Fursova, N. G. Kolosova, and L. E. Bakeeva, "Lipofuscin granule dynamics during development of age-related macular degeneration," Biochemistry, vol. 75, no. 2, pp. 130-138, 2010.

[10] V. B. Saprunova, M. A. Lelekova, N. G. Kolosova, and L. E. Bakeeva, "SkQ1 slows development of age-dependent destructive processes in retina and vascular layer of eyes of wistar and OXYS rats," Biochemistry, vol. 77, no. 6, pp. 648-658, 2012.

[11] Z. Zhao, Y. Chen, J. Wang et al., "Age-related retinopathy in NRF2-deficient mice," PLOS ONE, vol. 6, no. 4, Article ID e19456, 2011.

[12] H. Esfandiary, U. Chakravarthy, C. Patterson, I. Young, and A. E. Hughes, "Association study of detoxification genes in age related macular degeneration," The British Journal of Ophthalmology, vol. 89, no. 4, pp. 470-474, 2005.

[13] P. Hu, "Aryl hydrocarbon receptor deficiency causes dysregulated cellular matrix metabolism and age-related macular degeneration-like pathology," Proceedings of the National Academy of Sciences of the United States of America, vol. 110, no. 43, pp. E4069-E4078, 2013.

[14] J. P. Whitlock Jr., "Induction of cytochrome P4501A1," Annual Review of Pharmacology and Toxicology, vol. 39, pp. 103-125, 1999.

[15] Q. Ma, "Induction of CYP1A1: the AhR/DRE paradigm: transcription, receptor regulation, and expanding biological roles," Current Drug Metabolism, vol. 2, no. 2, pp. 149-164, 2001.

[16] R. L. Yeager, S. A. Reisman, L. M. Aleksunes, and C. D. Klaassen, "Introducing the "TCDD-inducible AhR-Nrf2 gene battery"," Toxicological Sciences, vol. 111, no. 2, pp. 238-246, 2009.

[17] K. Kato, K. Takahashi, S. Monzen et al., "Relationship between radiosensitivity and Nrf2 target gene expression in human hematopoietic stem cells," Radiation Research, vol. 174, no. 2, pp. 177-184, 2010.

[18] W. Jeong, M. Jun, and A. T. Kong, "Nrf2: a potential molecular target for cancer chemoprevention by natural compounds," Antioxidants and Redox Signaling, vol. 8, no. 1-2, pp. 99-106, 2006.

[19] J. D. Hayes, A. T. Dinkova-Kostova, and M. Mcmahon, "Crosstalk between transcription factors AhR and Nrf2: lessons for cancer chemoprevention from dioxin," Toxicological Sciences, vol. 111, no. 2, pp. 199-201, 2009.

[20] V. V. Neroev, M. M. Archipova, L. E. Bakeeva et al., "Mitochondria-targeted plastoquinone derivatives as tools to interrupt execution of the aging program. 4. Age-related eye disease. SkQ1 returns vision to blind animals," Biochemistry, vol. 73, no. 12, pp. 1317-1328, 2008.

[21] T. Nolan, R. E. Hands, and S. A. Bustin, "Quantification of mRNA using real-time RT-PCR,” Nature Protocols, vol. 1, no. 3, pp. 1559-1582, 2006.

[22] M. Nowak, W. Gnitecki, and P. Jurowski, "The role of retinal oxygen metabolism in origin of age-related macular degeneration (AMD)," Klinika Oczna, vol. 107, no. 10-12, pp. 715-718, 2005. 
[23] Y. Morel and R. Barouki, "Repression of gene expression by oxidative stress," Biochemical Journal, vol. 342, no. 3, pp. 481496, 1999.

[24] R. J. Roman, "P-450 metabolites of arachidonic acid in the control of cardiovascular function," Physiological Reviews, vol. 82, no. 1, pp. 131-185, 2002.

[25] D. Choudhary, I. Jansson, I. Stoilov, M. Sarfarazi, and J. B. Schenkman, "Metabolism of retinoids and arachidonic acid by human and mouse cytochrome P450 1B1," Drug Metabolism and Disposition, vol. 32, no. 8, pp. 840-847, 2004.

[26] D. Schwarz, P. Kisselev, S. S. Ericksen et al., "Arachidonic and eicosapentaenoic acid metabolism by human CYP1A1: highly stereoselective formation of $17(\mathrm{R}), 18(\mathrm{~S})$-epoxyeicosatetraenoic acid," Biochemical Pharmacology, vol. 67, no. 8, pp. 1445-1457, 2004.

[27] A. A. El-Sherbeni and A. O. El-Kadi, "Alterations in cytochrome P450-derived arachidonic acid metabolism during pressure overload-induced cardiac hypertrophy," Biochemical Pharmacology, vol. 87, no. 3, pp. 456-466, 2014.

[28] D. W. Nebert and T. P. Dalton, "The role of cytochrome P450 enzymes in endogenous signalling pathways and environmental carcinogenesis," Nature Reviews Cancer, vol. 6, no. 12, pp. 947960, 2006.

[29] A. B. Rifkind, "CYP1A in TCDD toxicity and in physiologywith particular reference to CYP dependent arachidonic acid metabolism and other endogenous substrates," Drug Metabolism Reviews, vol. 38, no. 1-2, pp. 291-335, 2006.

[30] J. M. Barnett, G. W. McCollum, and J. S. Penn, "Role of cytosolic phospholipase A2 in retinal neovascularization," Investigative Ophthalmology and Visual Science, vol. 51, no. 2, pp. 1136-1142, 2010.

[31] M. Al-Shabrawey, R. Mussell, K. Kahook et al., "Increased expression and activity of 12-lipoxygenase in oxygen-induced ischemic retinopathy and proliferative diabetic retinopathy: implications in retinal neovascularization," Diabetes, vol. 60, no. 2, pp. 614-624, 2011. 


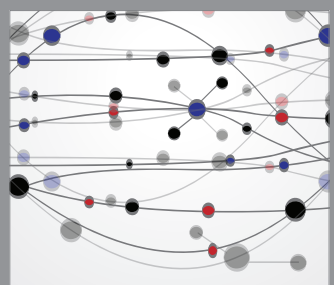

The Scientific World Journal
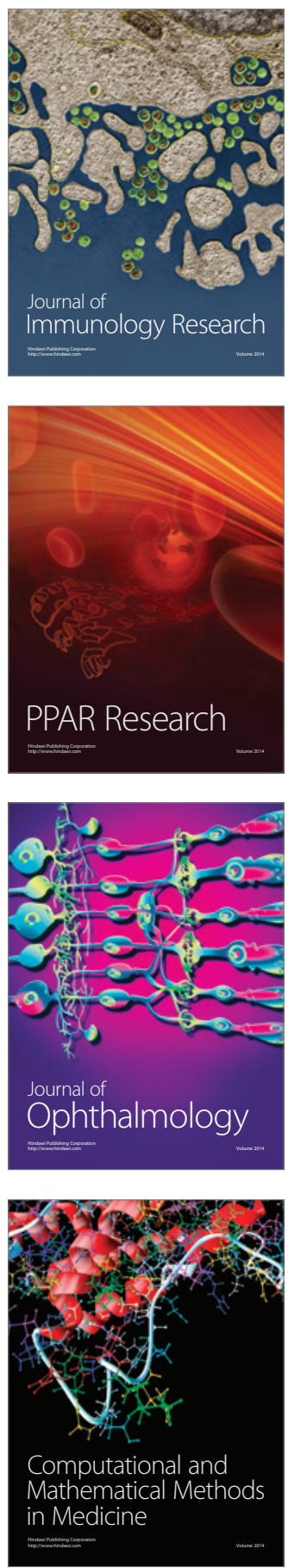

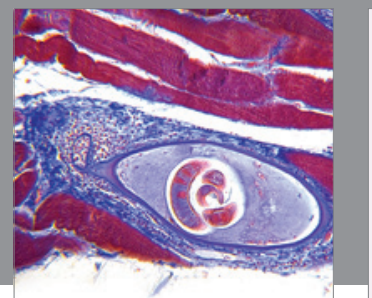

Gastroenterology

Research and Practice
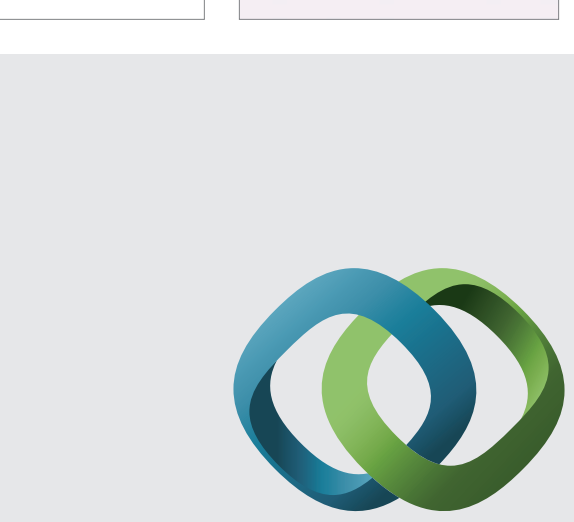

\section{Hindawi}

Submit your manuscripts at

http://www.hindawi.com
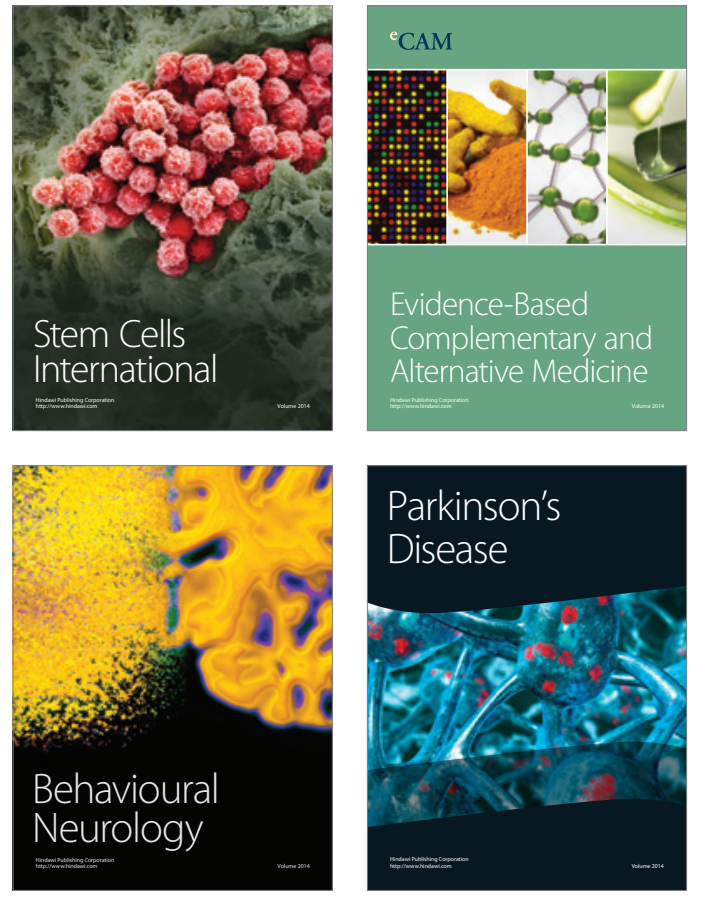
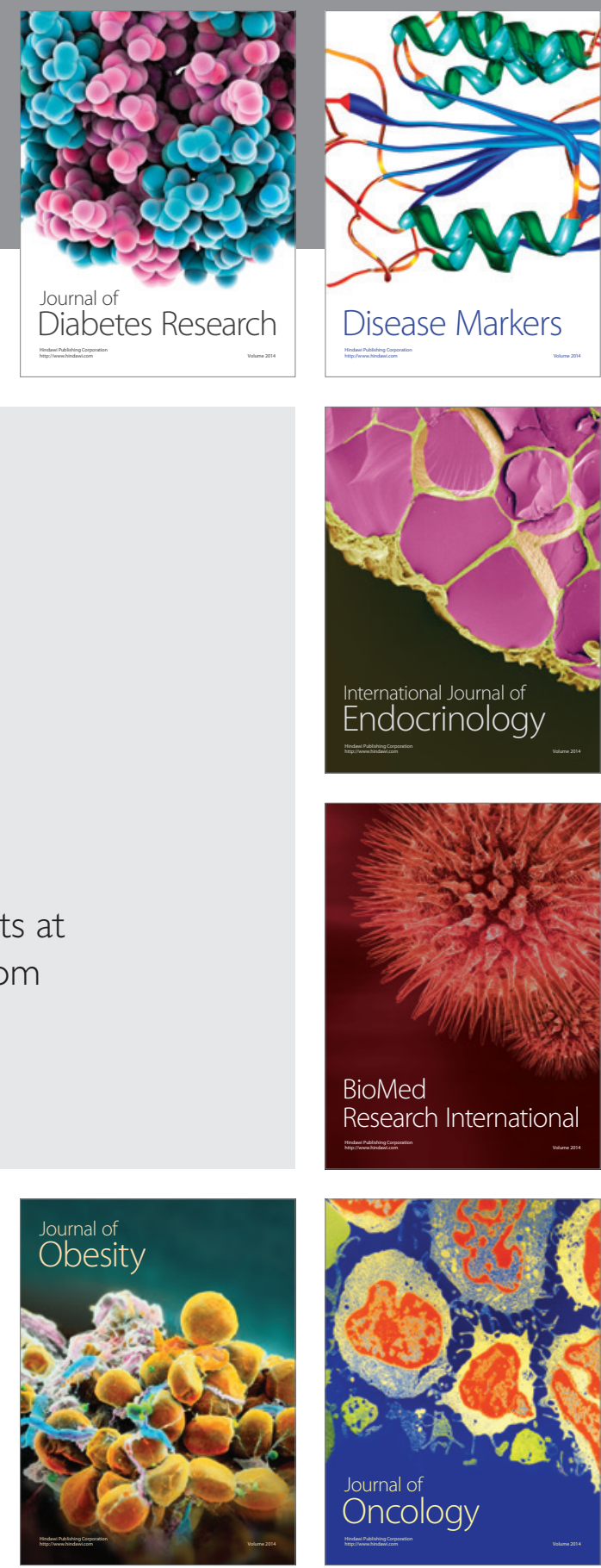

Disease Markers
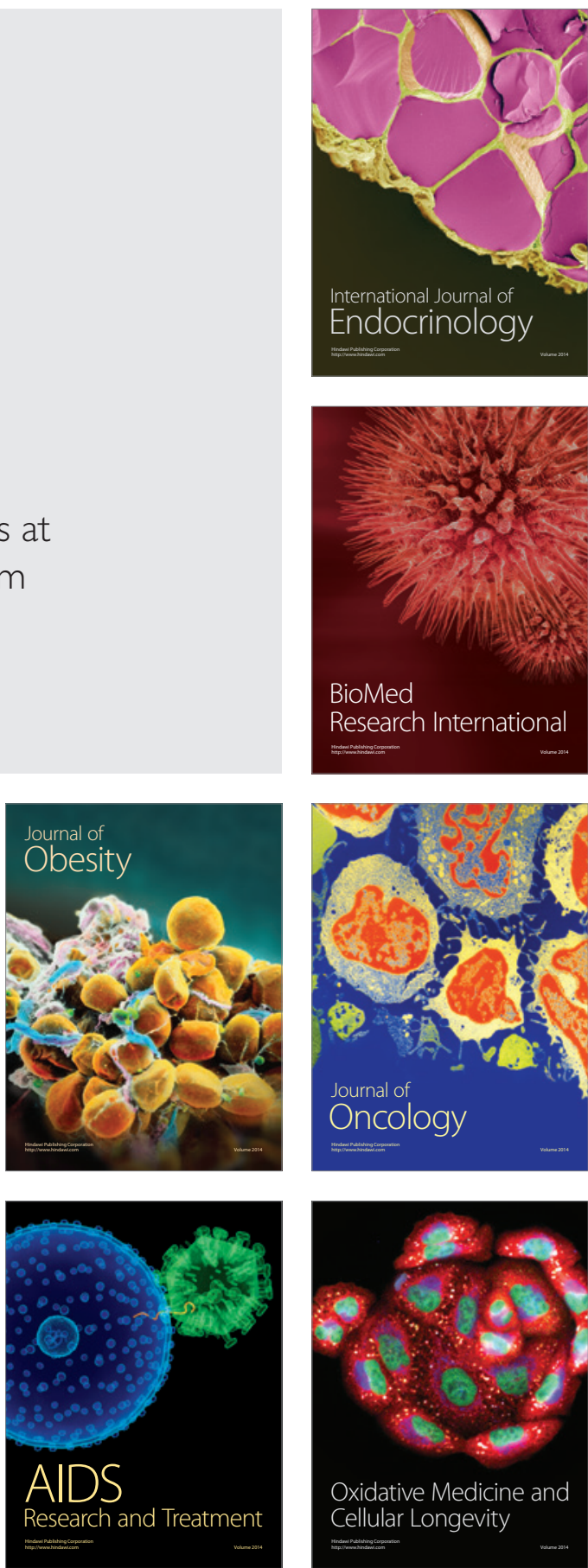